\title{
Unexpected HLA haplotype sharing in dizygotic twin pairs discordant for rheumatoid arthritis
}

\author{
Damini Jawaheer, Alexander J MacGregor, Peter K Gregersen, Alan J Silman, \\ William E R Ollier
}

\begin{abstract}
Dizygotic twins are generally believed to be no more genetically similar than sibs born from separate pregnancies. In the present study, a panel of 93 dizygotic twin pairs discordant for rheumatoid arthritis were typed for HLA-A, -B, -Cw, and -DR antigens. HLA haplotype sharing identical by descent between the twins showed a trend towards increased sharing of both HLA haplotypes; this increased sharing was statistically significant when the female/female twin pairs were considered separately. In contrast, the pattern of HLA haplotype sharing in sib pairs $(n=128)$ was consistent with a $1: 2: 1$ ratio of 2,1 , or 0 haplotypes shared. An analysis of 16 normal dizygotic twin pairs was consistent with these results raising the possibility that dizygotic twins in general are genetically more similar at the HLA complex than sibs born from separate pregnancies. ( $($ Med Genet 1996;33:1015-1018)
\end{abstract}

Key words: twin studies; HLA; dizygotic twins.

Twin studies have commonly been used as a research tool in clinical epidemiology and genetics to establish whether diseases such as insulin dependent diabetes (IDDM), rheumatoid arthritis (RA), systemic lupus erythematosus (SLE), and schizophrenia have a genetic component to their aetiology. Such studies involve comparisons of disease concordance figures between monozygotic (MZ) and dizygotic (DZ) twins, the basic principle being that a higher level of disease concordance among $M Z$ compared to $\mathrm{DZ}$ twins is evidence for a genetic contribution to the disease in question. Twin studies rely on a number of assumptions: (1) $\mathrm{MZ}$ twins are generally accepted as being genetically identical, originating from a single fertilised egg, (2) DZ twins are assumed to be no more genetically similar than sibs born from separate pregnancies, being the result of the independent fertilisation of two separate ova.

Given that the biological factors which underlie twinning are obscure, there remains some uncertainty regarding these assumptions. Although they acquire the same genomic DNA at fertilisation, $M Z$ twins may subsequently differ owing to independent somatic variation in the two genomes. Sources of variation include gene rearrangements in the immunoglobulin and $\mathrm{T}$ cell receptor loci, as well as the processes of $\mathrm{X}$ chromosome inactivation and genomic imprinting. ${ }^{1}$ In the present study, we report data which raise doubts about the
ARC Epidemiology

Research Unit,

University of

Manchester, Stopford

Building, Oxford

Road, Manchester

M13 9PT, UK

D Jawaheer

A J MacGregor

A J Silman

W E R Ollier

Division of Biology

and Human Genetics, Department of Medicine, North Shore University Hospital, 350 Community Drive, Manhasset, New York 11030, USA

D Jawaheer

P K Gregersen

Correspondence to: Dr Jawaheer, USA

Received 14 May 1996 Revised version accepted for publication 6 August 1996

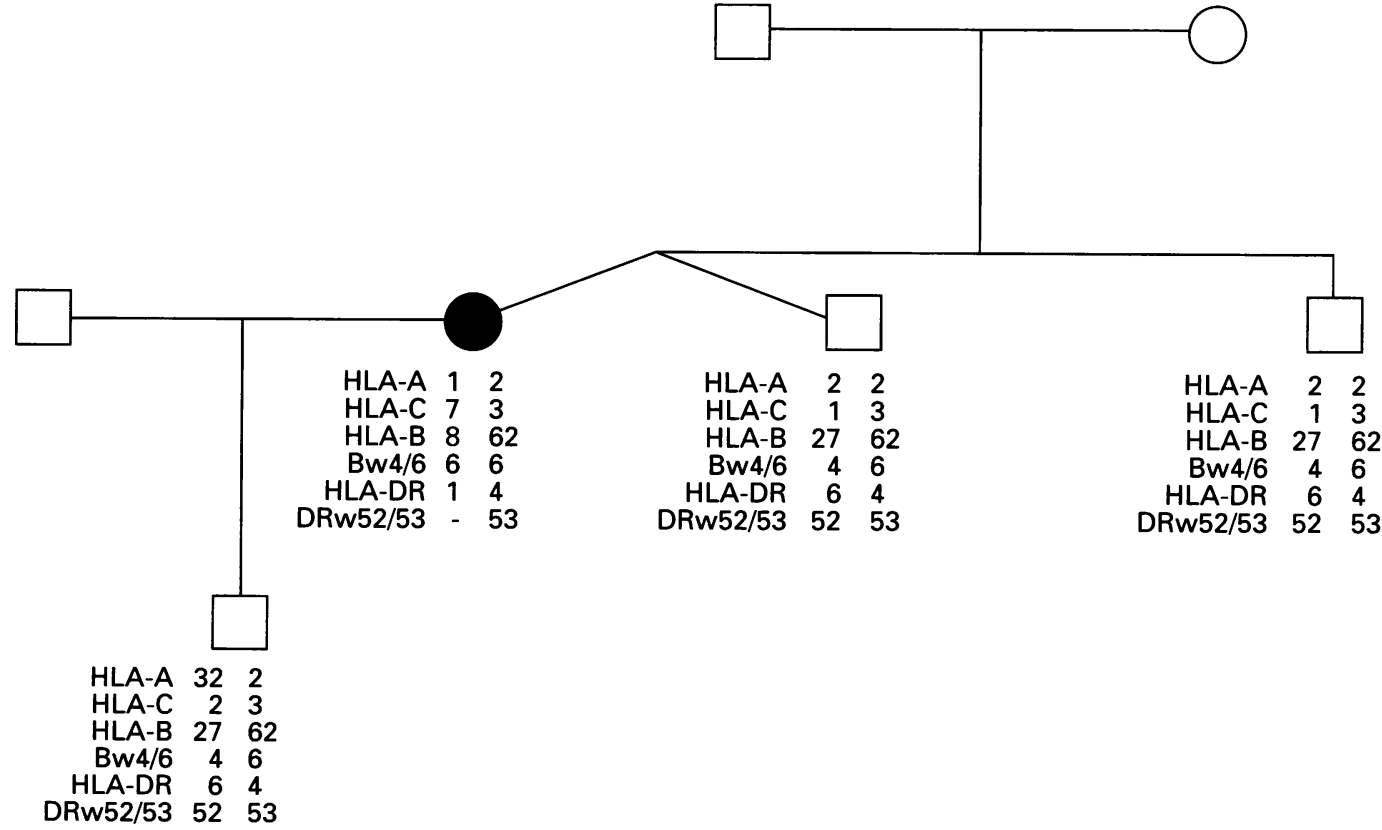

Figure 1 In this family, HLA typing results were available for the twin pair, one sib, and the son of the affected twin. The haplotype inherited from the affected twin by her son was first inferred. This was the A2Cw3B62Bw6DR1DRw53 haplotype. This same haplotype was shared among the two twins and their brother. 
Table 1 HLA sharing relationships among $D Z$ twin pairs

\begin{tabular}{llllll}
\hline No of HLA haplotypes shared & 2 & 1 & 0 & $\chi^{2}$ & $p$ \\
\hline$R A$ discordant twin pairs $(n=93)$ & & & & & \\
Observed No of twin pairs & 30 & 48 & 15 & & \\
Expected No of twin pairs & 23.25 & 46.5 & 23.25 & 4.94 & $<0.10$ \\
Non- $R A$ twin pairs $(n=16)$ & 11 & 3 & 2 & & \\
Observed No of twin pairs & 4 & 8 & 4 & 16.38 & $<0.001$ \\
Expected No of twin pairs & & & & & \\
\hline
\end{tabular}

Table 2 HLA sharing relationships among sib pairs

\begin{tabular}{llllll}
\hline No of HLA haplotypes shared & 2 & 1 & 0 & $\chi^{2}$ & $p$ \\
\hline$R A$ discordant sib pairs $(n=128)$ & 37 & 52 & 39 & & \\
$\begin{array}{l}\text { Observed No of pairs } \\
\text { Expected No of pairs }\end{array}$ & 32 & 64 & 32 & 4.56 & $<0.10$ \\
$\begin{array}{l}R A \text { discordant } D Z \text { twin/non-twin sib } \\
\text { pairs }(n=22)\end{array}$ & 7 & 12 & 3 & & \\
$\begin{array}{l}\text { Observed No of pairs } \\
\text { Expected No of pairs }\end{array}$ & 5.5 & 11 & 5.5 & 2.37 & $>0.25$ \\
\hline
\end{tabular}

Table 3 HLA haplotype sharing distribution in $R A$ discordant $D Z$ twin pairs compared to $R A$ discordant sib pairs

\begin{tabular}{llllll}
\hline No of HLA haplotypes shared & 2 & 1 & 0 & $\chi^{2}$ & $p$ \\
\hline No of DZ twin pairs $(\mathrm{n}=93)$ & 30 & 48 & 15 & & \\
No of sib pairs $(\mathrm{n}=128)$ & 37 & 52 & 39 & 6.17 & 0.046 \\
\hline
\end{tabular}

Table $4 H L A$ sharing relationships among $R A$ discordant $D Z$ twin pairs stratified by sex

\begin{tabular}{llllll}
\hline No of HLA haplotypes shared & 2 & 1 & 0 & $\chi^{2}$ & $p$ \\
\hline $\begin{array}{l}\text { Malelfemale twin pairs }(n=35)^{*} \\
\text { Observed No of pairs }\end{array}$ & 10 & 18 & 7 & & \\
$\begin{array}{l}\text { Expected No of pairs } \\
\text { Male/male DZ twin pairs }(n=15)\end{array}$ & 8.75 & 17.5 & 8.75 & 0.54 & $>0.75$ \\
$\begin{array}{l}\text { Observed No of pairs } \\
\text { Expected No of pairs }\end{array}$ & 3 & 9 & 3 & & \\
$\begin{array}{l}\text { Female/female DZ twin pairs }(n=43) \\
\text { Observed No of pairs }\end{array}$ & 17 & 7.5 & 3.75 & 0.60 & $>0.50$ \\
Expected No of pairs & 10.75 & 21.5 & 5 & & \\
\hline
\end{tabular}

*33 of the probands in this subgroup were female, and stratification based on the sex of the proband did not change the results shown.

second assumption, namely that $\mathrm{DZ}$ twins exhibit the same degree of genetic relatedness as sibs born from separate pregnancies. By assessing HLA haplotype sharing among DZ twins, we observed a surprising and significant increase in HLA identity among $93 \mathrm{DZ}$ twin pairs, despite the fact that these twins were discordant for an HLA associated illness, rheumatoid arthritis. Preliminary data suggest that this pattern of inheritance may also apply to normal $\mathrm{DZ}$ twins, perhaps more prominently in female/female pairs. These data have substantial implications with respect to the proper interpretation of $\mathrm{DZ}$ twin concordance data.

\section{Subjects and methods SUBJECTS}

The analysis was performed on 93 RA discordant $\mathrm{DZ}$ twin pairs (that is, only one twin from each pair had $R A$ ) out of an original population of $98 \mathrm{DZ}$ twin pairs. Five twin pairs were excluded from the current study because we were unable unambiguously to establish haplotype identity by descent among those pairs (see section on HLA typing below). The twins had been recruited as part of a nationwide twin study in the UK, conducted to investigate concordance figures in RA. The 93 RA dis- cordant DZ twin pairs were compared to two different control groups comprising (1) 128 RA discordant sib pairs from the ARC National Repository of RA multicase families, ${ }^{2}$ that is, one sib in each pair had RA, and (2) $22 \mathrm{sib}$ pairs, one of whom was an RA affected DZ twin from the subject group, and the other was a healthy non-twin sib. A second group of 16 DZ twin pairs, neither of whom had RA, were also analysed for HLA haplotype sharing.

\section{DISEASE CLASSIFICATION}

Subjects were classified as having RA based on the 1987 ACR criteria modified for epidemiological studies. ${ }^{3}$

\section{ZYGOSITY}

Zygosity of the twin pairs was established using DNA fingerprinting. ${ }^{4}$

\section{HLA TYPING}

All the subjects and controls, as well as their family members, were typed for HLA-A, -B, $-C w$, and -DR specificities by serology using the complement dependent microcytotoxicity assay. HLA-DR typing was confirmed by DNA analysis using PCR-SSO hybridisation. ${ }^{5}$ HLA haplotypes were established within each family, ensuring that inheritance of these haplotypes in the families was consistent (see fig 1 for example). Thus, it was possible to determine haplotype sharing (identity by descent) for each twin pair, despite the lack of parental DNA for most twins. As noted above, five twin pairs were excluded from our final analysis. Four of the excluded pairs were HLA identical and one pair shared no HLA haplotype, and there was a possibility that haplotypes shared in these cases were identical by state (IBS) only and not identical by descent (IBD).

\section{STATISTICAL ANALYSIS}

Within each group of twin or sib pairs, the number of pairs sharing 2,1 , or 0 HLA haplotypes identical by descent was determined. A $\chi^{2}$ test ( 2 degrees of freedom) was then used to determine whether the pattern of HLA haplotype sharing in each group differed significantly from the expected $1: 2: 1$ ratio in sibs. The distribution of HLA haplotype sharing among the $93 \mathrm{DZ}$ twin pairs was also compared to that in the $128 \mathrm{sib}$ pairs using a $\chi^{2}$ test. $\chi^{2}$ values were considered to be significant when $\mathrm{p}<0.05$. The subject group was subsequently subdivided in terms of gender and the analysis was repeated within each subgroup.

\section{Results}

The $\chi^{2}$ test results for the different twin and sib groups are summarised in tables 1 and 2 . As shown in table 1, an increased number of twin pairs shared two HLA haplotypes compared to the expected numbers among the 93 RA discordant twin pairs $(p<0.1)$. In a second group of $16 \mathrm{DZ}$ twin pairs without RA, this 
trend was statistically significant $(p<0.001)$ There was no significant difference between observed and expected numbers in the two other groups, that is, the $22 \mathrm{RA}$ discordant sib pairs and the $128 \mathrm{RA}$ discordant sib pairs (table 2). Comparison of the haplotype distribution in the $93 \mathrm{DZ}$ twin pairs with the 128 sib pairs showed that there is a statistically significant distortion in the distribution of haplotypes in the twin pairs $(p=0.046)$ (table 3$)$.

When the analysis was performed on the subgroups of male/female, male/male, and female/female twin pairs, only the latter subgroup showed a statistically significant increase in sharing of HLA haplotypes compared to expected numbers $(\mathrm{p}<0.025)$ (table 4). The number of male/male twin pairs was too small for any conclusions to be drawn. In the male/ female subgroup, the expected 1:2:1 ratio of 2,1 , or 0 HLA haplotype sharing was observed. When haplotype sharing was assessed in the corresponding subgroups among the $128 \mathrm{sib}$ pairs, all three subgroups showed a 1:2:1 sharing of 2,1 , or 0 haplotypes (data not shown).

\section{Discussion}

Our observations suggest that DZ twins exhibit a higher level of genetic similarity in the HLA region than expected. This is not observed in sibs even within the same families. In the twins with RA, only one twin in each pair suffered from rheumatoid arthritis, and thus it is not likely that the increased sharing of HLA haplotypes is related to the disease. Indeed, one might well expect a decrease in HLA sharing among RA discordant sib pairs since HLA is known to predispose to this disease. Increased HLA haplotype sharing among the 16 RA free $\mathrm{DZ}$ twin pairs indicates that the phenomenon is probably a feature of $\mathrm{DZ}$ twinning itself. One intriguing aspect of the increased haplotype sharing is that, when the twin pairs were stratified in terms of sex, only the female/female pairs displayed the phenomenon. No conclusions could be drawn about the male/male pairs as the numbers were too small $(n=15)$. Moreover, the male/female twin pairs $(n=35)$ showed a 1:2:1 ratio of 2,1 , or 0 HLA haplotype sharing as observed for sibs. Interestingly, a recent study of a population of normal Danish DZ twins showed that twin pairs of the same sex shared HLA haplotypes more often than did twins of different $\operatorname{sex},{ }^{6}$ thus supporting our observations. The departure from 1:2:1 segregation among the same sexed twin pairs was statistically significant in that study $(\mathrm{p}<0.05)$.

Interestingly, of the five twin pairs we excluded from our analysis, four shared both of their HLA haplotypes and one shared none, though this sharing could be by state (IBS) and not by descent (IBD). HLA haplotypes are so polymorphic that the chance of the twins sharing a haplotype IBS and not IBD is very low. Bearing this in mind, if we included these five twin pairs in our analysis, the distortion in distribution of haplotype sharing among 98 twin pairs would be statistically significant $\left(\chi^{2}=7.37, \mathrm{p}<0.025\right)$. To support our results, we coded each of 177 haplotypes as an "allele" and performed an IBS analysis on the data on the 93 twin pairs using the GAS (Genetic Analysis System, Version 2.0) software. The increase in haplotype sharing IBS was statistically significant $(p=0.03$, one tailed test), even though the five twin pairs sharing HLA haplotypes were not included. The IBS results for the subgroups stratified by sex were similar to those shown in table 4 .

The increase in haplotype sharing among $\mathrm{DZ}$ twins may relate to either maternal-fetal or fetal-fetal incompatibility. The maternal immune system regards the presence of the fetus as a graft expressing self as well as foreign antigens encoded by paternally inherited genes. ${ }^{7}$ There is some doubt as to whether HLA incompatibility between mother and fetus assists pregnancy or may lead to abortion. In experimental animals, fetomaternal incompatibility at the major histocompatibility complex has been found to be increased in primary abortions. ${ }^{8}$ In humans, however, inherited paternal HLA alleles not shared by the mother have been shown to be beneficial to the survival of the fetus, perhaps by mechanisms involving immune suppression. ${ }^{9}$ In rheumatoid arthritis patients, pregnancy is known to induce remission of this autoimmune disease, and it has recently been shown that this phenomenon is dependent on the fetus having inherited paternal HLA haplotypes not shared by the mother. ${ }^{10}$ Whatever the mechanism, if survival as a member of a $\mathrm{DZ}$ twin pair depends in part upon paternally inherited HLA haplotypes, it would be predicted that $\mathrm{DZ}$ twins would share paternal haplotypes more frequently than expected. Clearly, such a selection mechanism does not operate on singleton pregnancies, since no bias toward HLA sharing occurs in sib pairs (table 2). In this study, we have not determined whether there is an overall bias towards sharing of maternal or paternal haplotypes. Identity by descent was determined in most twin pairs by establishing haplotypes from information on the sibs and children of the $\mathrm{DZ}$ twins. The issue of parental bias in haplotype sharing will be addressed in an additional population of $\mathrm{DZ}$ twin pairs.

The increased HLA haplotype sharing among $\mathrm{DZ}$ twins may also relate to the phenomenon of "the vanishing twin". In a study of 325 twin conceptions, ${ }^{11}$ there were 125 cases where one twin from a pair was resorbed so that only one of the twins was present at birth; there were also 139 cases where both twins were lost. The reason why this phenomenon occurs is not known, but we speculate that it may be related to the patterns of HLA haplotype sharing between the twins and to maternal-fetal or fetal-fetal incompatibility. Moreover, we have yet to determine whether the HLA complex is the only region in the genome shared by the twins. Other regions may also be shared that play a role in the survival of twins.

We cannot explain why increased HLA haplotype sharing appears to be restricted to female/female twin pairs in our data set. There is evidence that fetal-maternal HLA 
relationship may be less important for fetal survival in the case of a male fetus. ${ }^{12}$ The reason for this is at present unclear and it is difficult to account for all these observations given the current state of knowledge. It will be important finally to establish the pattern of HLA sharing in male/male $\mathrm{DZ}$ twins in order to gain further insight into this problem.

Our finding that $\mathrm{DZ}$ twins are genetically more similar than sibs born from separate pregnancies has important implications in the field of twin studies. It implies that the estimation of the extent of the genetic component of a disease based on disease concordance figures should be interpreted with caution.

We would like to thank Sarah Bamber for collecting blood samples from the twins, and David Carthy and Ayar Farhan for processing the samples. HLA class I typing by serology was performed by staff of the North West Regional Tissue Typing laboratory, St Mary's Hospital in Manchester (UK). We also thank Nina Kohn for her comments about the statistical analyses.

1 Gregersen PK. Discordance for autoimmunity in monozygotic twins. Are identical twins really identical? Arthritis Rheum 1993;36:1185-92.
2 Hay EM, Ollier WER, Silman AJ. The Arthritis and Rheumatism Council's National Family Material Repository. $B$ f Rheumatol 1993;32:443-4.

3 MacGregor AJ, Bamber S, Silman AJ. A comparison of the performance of different methods of disease classification for rheumatoid arthritis. Results from the analysis from nationwide twin study. $\mathcal{F}$ Rheumatol 1994;21:1420-6.

4 Silman AJ, MacGregor AJ, Thomson W, et al. Twin concordance rates for rheumatoid arthritis: results from a nationwide study. Br F Rheumatol 1993;32:903-7.

5 Thomson W, Ollier WER. Non-radioactive ASO typing for class II: the way forward. Eur $\mathcal{f}$ Immunogenet 1992;19: class II:

6 Staub Nielsen L, Eiberg H, Fenger K, Mohr J. An MHC (HLA-A, -B, C2, BF, HLA-DR, GLO1) haplotype study of 497 Danish normal families with 1970 children in cluding 97 twin pairs. Tissue Antigens 1990;36:141-8.

7 Wegmann TG, Lin H, Guilbert L, Mosmann TR. B directional cytokine interactions in the maternal-fetal relationship: is successful pregnancy a $\mathrm{T}_{\mathrm{H}} 2$ phenomenon? Immunol Today 1993;14:353-6.

8 Laitinen T, Koskimies S, Westman P. Foeto-maternal compatibility in HLA-DR, -DQ and -DP loci in Finnish couples suffering from recurrent spontaneous abortions. Eur f Immunogenet 1993;20:249-58.

9 Ober C, Elias S, Kostyu DD, Hauck WW. Decreased fecundability in Hutterite couples sharing HLA-DR. $A m \mathcal{F}$ Hum Genet 1992;50:6-14.

10 Nelson JL, Hughes KA, Smith AG, Nisperos BB, Branchaud AM, Hansen JA. Maternal-fetal disparity in HLA class II alloantigens and the pregnancy-induced amelioration of rheumatoid arthritis. $N$ Engl $\mathcal{F}$ Med 1993;329:500-1.

11 Landy HJ, Keith L, Keith D. The vanishing twin. Acta Genet Med Gemellol (Roma) 1982;31:179-94.

12 Radvany RM, Vaisrub N, Ober C, Patel KM, Hecht F. The human sex ratio: increase in first-born males to parents with shared HLA-DR antigens. Tissue Antigens 1987;29: 34-42. 